\title{
Central and Basolateral Amygdala Neurons Crash the Aversive Conditioning Party: Theoretical Comment on Rorick-Kehn and Steinmetz (2005)
}

\author{
Stephen Maren \\ University of Michigan, Ann Arbor
}

\begin{abstract}
Rorick-Kehn and Steinmetz (2005) report that neurons in the central and basolateral nuclei of the amygdala exhibit learning-related spike firing to conditional stimuli associated with shock in 3 different aversive conditioning paradigms: eyeblink conditioning, fear conditioning, and signaled avoidance conditioning. Central nucleus neurons responded in all 3 tasks, whereas basolateral nucleus neurons were more activated by fear and avoidance conditioning. These results reveal that amygdala neurons are differentially engaged by aversive conditioning, but questions remain concerning the associative basis and functional role for these unit responses.
\end{abstract}

Keywords: avoidance, Pavlovian conditioning, amygdala, fear, rat

Considerable interest has emerged in recent years concerning the involvement of the amygdala in emotional learning and memory (Davis, 1992; Fanselow \& Poulos, 2005; LeDoux, 2000; Maren, 2001; McGaugh, 2004). Much of the work in this area has focused on the contribution of the amygdala to Pavlovian fear conditioning, a form of aversive learning in which an innocuous conditional stimulus (CS), such as a tone, signals an unconditional stimulus (US), such as a footshock. The amygdala is anatomically and functionally heterogeneous, consisting of at least nine different nuclei, only some of which are involved in fear conditioning. In this regard, several studies have revealed an important role for the lateral; central; and, in some cases, basolateral amygdaloid nuclei in the acquisition and expression of fear conditioned responses. Nonetheless, there is debate concerning the precise role of these nuclei in the genesis of learned fear (Killcross, Robbins, \& Everitt, 1997; Pare, Quirk, \& Ledoux, 2004).

Rorick-Kehn and Steinmetz (2005) have attempted to clarify the role of two of the amygdaloid nuclei, the central and basolateral nuclei, in aversive conditioning using single-unit recording techniques in three different behavioral tasks in rats. Until this study, most electrophysiological work on the amygdala focused on the contribution of the lateral nucleus to Pavlovian fear conditioning (Maren \& Quirk, 2004). Indeed, the lateral nucleus is believed to be a major sensory gateway into the amygdala. Lateral nucleus neurons exhibit short-latency spikes (10-12 ms) to auditory stimuli that greatly increase in frequency after fear conditioning (Collins \& Pare, 2000; Maren, 2000; Quirk, Armony, \& LeDoux, 1997; Quirk, Repa, \& LeDoux, 1995; Repa et al., 2001). By all accounts these increases in short-latency spike firing in the lateral nucleus are due to an association between the CS and US (i.e., the associative memory) and not the arousal of fear that the conditioning

Correspondence concerning this article should be addressed to Stephen Maren, Department of Psychology and Neuroscience Program, University of Michigan, 530 Church Street, Ann Arbor, MI 48109-1043. E-mail: maren@umich.edu procedures engender (Goosens, Hobin, \& Maren, 2003). And although central nucleus neurons have also been examined during fear conditioning in rabbits (Applegate, Frysinger, Kapp, \& Gallagher, 1982; Pascoe \& Kapp, 1985), there has been little work on this nucleus in the rat and no work directly comparing the activity of different amygdaloid nuclei during different aversive conditioning tasks (but see Muramoto, Ono, Nishijo, \& Fukuda, 1993; Uwano, Nishijo, Ono, \& Tamure, 1995).

Simultaneously recording neuronal activity in the central and basolateral nuclei of rats, Rorick-Kehn and Steinmetz (2005) report that auditory CSs that have been associated with shock during eyeblink conditioning (Experiment 1), fear conditioning (Experiment 2), or signaled bar-press avoidance conditioning (Experiment 3) evoke a greater neuronal response (i.e., a greater proportion of cells exhibiting a significant increase in spike firing during the tone relative to the period before tone onset) in central and basolateral neurons after conditioning. In the case of eyeblink conditioning, it could be concluded with some certainty that this pattern of activity was related to the associative contingency of the task. That is, neuronal responses were greater on trials associated with a conditional response and a greater proportion of neurons were active in animals receiving paired as compared to unpaired training, at least later in conditioning. This was particularly true for central nucleus neurons, although there was also evidence that basolateral nucleus neurons encoded the CS-US association. This is an important observation insofar as the amygdala plays an important role in the acquisition of eyeblink conditioning (Christian \& Thompson, 2003; Lee \& Kim, 2004), and earlier studies of spike firing during eyeblink conditioning found little evidence of associative plasticity in the amygdala (Richardson \& Thompson, 1984).

In the fear conditioning and avoidance conditioning paradigms, however, the pattern of spike firing in the basolateral and central nuclei was not as clearly associative in nature. In the fear conditioning task, footshock exposure in both the paired and unpaired groups increased the proportion of neurons responsive to the tone 
$\mathrm{CS}$, as well as yielding equivalent levels of conditional freezing in the two groups of animals. This pattern of neural activation persisted in unpaired animals tested to the CS outside of the conditioning context; in this case, rats froze considerably less to the CS (indicating that fear to the conditioning context was responsible for the freezing behavior generated by the unpaired procedure) but maintained elevated neural responsiveness to the CS. Similarly, neuronal activity in the central and basolateral nuclei was elevated by both the paired and unpaired procedures in the avoidance task, and basolateral neurons were actually more active in the unpaired group. Unlike earlier reports that identified associative factors underlying lateral nucleus spike firing during Pavlovian fear conditioning (Goosens et al., 2003), nonassociative sensitization may have accounted for the elevations in spike firing reported by Rorick-Kehn and Steinmetz (2005). Nonetheless, elevated activity during avoidance conditioning in both the central and basolateral nucleus was not evident in rats that did not learn the avoidance response, suggesting that neural activation in the central and basolateral nuclei may have been related to the mnemonic demands of the task, not merely exposure to aversive stimuli.

On the basis of these results, the primary conclusions made by Rorick-Kehn and Steinmetz (2005) were that the proportion of central and basolateral nucleus neurons activated by an auditory CS increases after the CS comes to be associated with an aversive US, that the degree of neuronal activation in the amygdala may reflect the aversiveness of the US, and that the activity of basolateral nucleus neurons appears to be more strongly modulated by instrumental training. These data are consistent with some models of amygdala function (Amorapanth, LeDoux, \& Nader, 2000; Killcross et al., 1997), and support recent claims for an associative role for the central nucleus in aversive conditioning (Pare et al., 2004). The Rorick-Kehn and Steinmetz (2005) results shed new light on the involvement of two relatively understudied amygdaloid nuclei in aversive conditioning, and suggest that there is more distributed associative coding within the amygdala than previously recognized. Nonetheless, there are some unexpected results and inconsistencies with published reports that deserve further attention.

\section{To Associate or Not to Associate. . .}

One surprising outcome of the Rorick-Kehn and Steinmetz (2005) report is the extent to which unpaired training in both the fear conditioning and avoidance conditioning paradigms elevated the proportion of neurons responsive to the auditory CS. In the fear conditioning experiment, the levels of activation in the amygdala were indistinguishable in paired and unpaired animals. It is interesting that mere shock exposure did not appear to sensitize amygdaloid unit activity, insofar as animals that did not learn the avoidance conditioned response did not exhibit increased amygdala activation (although there may have been other behavioral differences between these populations that accounted for the different unit activity profiles). In contrast, it has typically been reported, at least in recordings made from the lateral nucleus, that paired training increases CS-elicited spike firing above and beyond that produced by unpaired training in either a within-subject design (Collins \& Pare, 2000; Goosens et al., 2003; Quirk et al., 1995; Repa et al., 2001) or a between-subjects design (Maren, 2000). For example, it has been reported that spike firing increases in the same subjects during paired training relative to an earlier session of unpaired training (Quirk et al., 1995; Repa et al., 2001) or to a $\mathrm{CS}+$ relative to a $\mathrm{CS}-$ in a discriminative conditioning procedure (Collins \& Pare, 2000; Goosens et al., 2003), It is unlikely that the location of the recordings (basolateral-central vs. lateral) yielded this pattern, insofar as Kapp and colleagues have found associative spike firing in the central nucleus during discriminative fear conditioning in rabbits (Applegate et al., 1982; Pascoe \& Kapp, 1985) and Gabriel and colleagues found associative plasticity in the basolateral nucleus during avoidance conditioning in rabbits (Maren, Poremba, \& Gabriel, 1991).

What accounts for the sensitization of basolateral and central activity in response to footshock in the Rorick-Kehn and Steinmetz (2005) study? One possibility is that the analytical methods used by Rorick-Kehn and Steinmetz failed to detect critical differences in firing rate within single amygdala neurons. Because the behavioral paradigms used by these authors (with the exception of fear conditioning) required recording neuronal activity over several sessions (days), they were limited in their ability to characterize spike firing in the same neurons across sessions. Rather, they treated neurons recorded in each behavioral session as an independent population and then described task-related activity in terms of the proportion of neurons in that population that were responsive to the CS (defined by any increase in spike firing to the CS that was roughly two standard deviations above the pre-CS baseline) in any given session. Although this type of analysis yields information concerning the number of neurons engaged by the behavioral procedures, it does not quantify the magnitude of firing rate changes within that population of neurons (see Figure 1).

Although studies of lateral nucleus spike firing have revealed increases in the proportion of neurons that respond to the CS after conditioning (Quirk et al., 1995), they have also shown that conditioning quantitatively increases the neuronal firing rate among those cells that are activated by the CS (Collins \& Pare, 2000; Goosens et al., 2003; Maren, 2000; Repa et al., 2001). Analysis of the associative properties of lateral nucleus spike firing has demonstrated that the firing rate of individual neurons is coupled to the associative history of the CS, and is not a mere consequence of US exposure or fear behavior (Goosens et al., 2003). Because this analysis was conducted using a discriminative conditioning procedure (within-subject), it is insensitive to changes in the overall population of CS-responsive neurons. LeDoux and colleagues (Repa et al., 2001) have reported similar results. Hence, changes in synaptic strength of auditory afferents onto amygdaloid neurons (Blair, Schafe, Bauer, Rodrigues, \& LeDoux, 2001; Maren, 1999a), which are regulated by associative contingencies (Bauer, LeDoux, \& Nader, 2001), may regulate firing rate in amygdaloid neurons (Buzsaki et al., 2002), and this, in turn, may encode critical aspects of the CS-US contingency.

\section{It's About Time. . .}

Another important difference between the Rorick-Kehn and Steinmetz (2005) study and earlier work is the use of relatively long temporal bins to analyze post-CS spike firing. In the fear conditioning and avoidance conditioning experiments, the bin width was $1,000 \mathrm{~ms}$, which is $20-100$ times longer than the bin widths previously used to characterize lateral nucleus spike firing during fear conditioning. This is an important issue, because the 


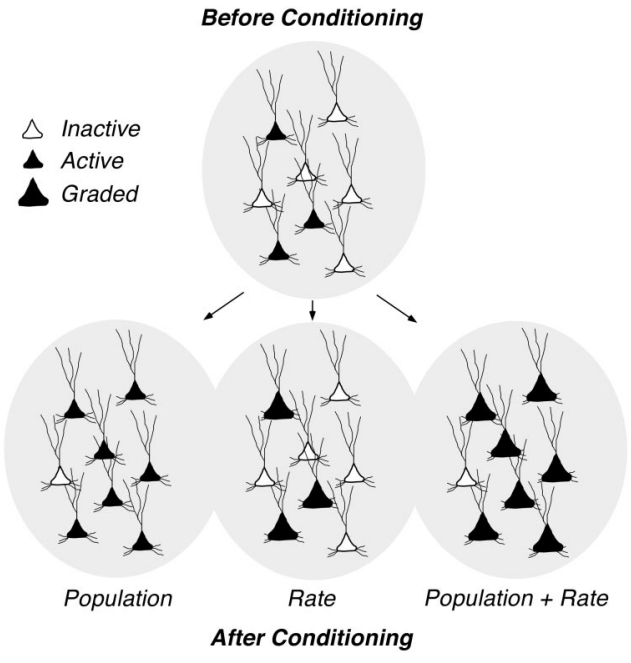

Figure 1. Hypothetical model of information coding within a population of neurons during aversive conditioning. Prior to conditioning (top), conditional stimuli (CSs) activate a subset of the neuronal population. That is, CSs increase firing rate in a particular neuron above some threshold relative to the pre-CS baseline (CSs also produce graded activity, reflected in diverse firing rates of particular neurons to the CS, but this is omitted for simplicity in this panel). After conditioning (bottom panels), the CS may recruit more active neurons that exceed this firing rate threshold (population code), yield graded elevations in the suprathreshold firing rate (rate code), or both (population + rate code). The neuronal analysis performed by Rorick-Kehn and Steinmetz (2005) focuses on population coding and does not address quantitative changes in firing rate within the population of CS-responsive neurons (rate or population + rate codes).

monosynaptic projections to the amygdala that carry auditory information from the acoustic thalamus generate short-latency spike firing from 10 to $100 \mathrm{~ms}$ after CS onset. The majority of studies analyzing the profile of CS-evoked firing in the amygdala have focused on this brief post-CS interval, and many have focused on spike firing occurring within the first $50 \mathrm{~ms}$ after onset of the CS (Maren, 2000; Maren et al., 1991; Quirk et al., 1995; Repa et al., 2001). Hence, the use of long-duration bins by Rorick-Kehn and Steinmetz (Experiments 2 and 3) diluted changes in shortlatency $(0-50 \mathrm{~ms})$ spike firing in the amygdala that have been at the core of the analyses characterizing associative plasticity of lateral nucleus firing in earlier studies. This is not to say that short-latency plasticity did not occur in the Rorick-Kehn and Steinmetz study; additional analyses using shorter bin widths may in fact reveal such plasticity (although there was not a significant difference in the first 70 -ms bin in the eyeblink conditioning experiment). Indeed, other work has revealed short-latency, associative activity in the central (Applegate et al., 1982; Pascoe \& Kapp, 1985) and basolateral nuclei (Maren et al., 1991) after aversive conditioning.

Although short-latency spike firing in the Rorick-Kehn and Steinmetz (2005) study was not reported, it is interesting to consider the possibility that the long-latency activation (1-4 s) of central and basolateral neurons they reported might encode the emotional state of the animal (whether a consequence of associative or nonassociative factors), and that this population code might convey different information than the information carried by the short-latency firing rate code of individual amygdaloid neurons. Indeed, there have been recent suggestions that the excitability of lateral amygdaloid neurons, reflected in their spontaneous firing, might be regulated by shock exposure (Goosens et al., 2003; Pelletier, Likhtik, Filali, \& Pare, 2005). It is conceivable that the recruitment of CS-responsive cells in the Rorick-Kehn and Steinmetz study in the fear and avoidance conditioning experiments reflects the nonspecific increase in neuronal excitability caused by strong footshocks (a product of nonassociative sensitization), rather than increases specific to the associative contingency.

\section{Reaction or Action?}

In the Rorick-Kehn and Steinmetz (2005) study, basolateral neurons exhibited differential activation in the paired and unpaired conditions in the avoidance task (albeit with greater activation in the unpaired compared to paired groups), and central neurons differentiated between paired and unpaired conditions much more than basolateral neurons during eyeblink conditioning. RorickKehn and Steinmetz therefore argue that the central nucleus may have a role in associating CSs and USs to yield conditional fear responses (reaction), whereas the basolateral nucleus may be more intimately involved in representing the incentive properties of the US necessary for avoidance responding (action). However, this dichotomy is not so clear-cut insofar as basolateral neurons did in fact respond to the associative contingencies during eyeblink conditioning, and central nucleus neurons responded to CSs in rats that learned the avoidance response, but not in rats that did not learn the avoidance response. And there is considerable evidence that the lateral nucleus, which is upstream of both the central nucleus and basolateral nucleus, exhibits associative plasticity during fear conditioning (Maren \& Quirk, 2004).

Nonetheless, it is not inconceivable that the central nucleus encodes CS-US associations during aversive conditioning. This was originally suggested by Kapp and colleagues (Kapp, Frysinger, Gallagher, \& Haselton, 1979) and finds recent support in a number of studies (Goosens \& Maren, 2003; Pare et al., 2004). What remains unclear is whether the central nucleus can encode CS-US associations independently of the lateral or basolateral nuclei. The ability of rats with basolateral complex lesions (lesions that include the lateral and basolateral nuclei) to acquire conditional fear responses (Gale et al., 2004; Maren, 1999b) suggests that the central nucleus may mediate independent associative functions. Yet, whether neuronal coding in the central nucleus requires afferent input from the basolateral complex requires further investigation.

Animals with central nucleus lesions can acquire instrumental avoidance responses, whereas animals with basolateral nucleus lesions cannot (Amorapanth et al., 2000; Killcross et al., 1997; Poremba \& Gabriel, 1999). Thus, the activation of basolateral neurons during avoidance conditioning (Maren et al., 1991; Muramoto et al., 1993; Rorick-Kehn \& Steinmetz, 2005), might be interpreted as a role for the basolateral nucleus in encoding the instrumental (action-outcome) contingency necessary for avoidance performance (Cardinal, Parkinson, Hall, \& Everitt, 2002; Everitt, Cardinal, Parkinson, \& Robbins, 2003). Yet, although some reports have suggested that the basolateral nucleus is not essential for Pavlovian conditioning (Killcross et al., 1997; Nader, Majidishad, Amorapanth, \& LeDoux, 2001), others have indicated 
that it might be involved (Goosens \& Maren, 2001). Consistent with two-process models of avoidance learning, it has been suggested that basolateral neurons may operate in the coding of Pavlovian associations that precede avoidance learning (Amorapanth et al., 2000; Maren, 1998). Thus, although neurons in the basolateral nucleus become active during instrumental learning (Maren et al., 1991; Muramoto et al., 1993; Rorick-Kehn \& Steinmetz, 2005), this activity may reflect the encoding of Pavlovian associations. The relatively greater activation of basolateral neurons early in avoidance conditioning (Maren et al., 1991; Rorick-Kehn \& Steinmetz, 2005), when Pavlovian associations are learned, supports this claim.

\section{Conclusions}

Rorick-Kehn and Stenimetz (2005) report interesting new results concerning the activity of single central and basolateral amygdala neurons during three aversive conditioning tasks. Few studies have directly contrasted these two amygdaloid nuclei, and it is now clear that distinct patterns of responding emerge in these regions during emotional learning. This suggests some degree of functional heterogeneity in the amygdaloid network and reveals potential dissociations in the nature of the representations encoded by amygdaloid nuclei. This is an important step in defining the nature and content of the neural code for aversive memory that is represented by the spike activity of amygdaloid neurons.

\section{References}

Amorapanth, P., LeDoux, J. E., \& Nader, K. (2000). Different lateral amygdala outputs mediate reactions and actions elicited by a feararousing stimulus. Nature Neuroscience, 3, 74-79.

Applegate, C. D., Frysinger, R. C., Kapp, B. S., \& Gallagher, M. (1982). Multiple unit activity recorded from amygdala central nucleus during Pavlovian heart rate conditioning in rabbit. Brain Research, 238, 457462.

Bauer, E. P., LeDoux, J. E., \& Nader, K. (2001). Fear conditioning and LTP in the lateral amygdala are sensitive to the same stimulus contingencies. Nature Neuroscience, 4, 687-688.

Blair, H. T., Schafe, G. E., Bauer, E. P., Rodrigues, S. M., \& LeDoux, J. E. (2001). Synaptic plasticity in the lateral amygdala: A cellular hypothesis of fear conditioning. Learning \& Memory, 8, 229-242.

Buzsaki, G., Csicsvari, J., Dragoi, G., Harris, K., Henze, D., \& Hirase, H. (2002). Homeostatic maintenance of neuronal excitability by burst discharges in vivo. Cerebral Cortex, 12, 893-899.

Cardinal, R. N., Parkinson, J. A., Hall, J., \& Everitt, B. J. (2002). Emotion and motivation: The role of the amygdala, ventral striatum, and prefrontal cortex. Neuroscience \& Biobehavioral Reviews, 26, 321-352.

Christian, K. M., \& Thompson, R. F. (2003). Neural substrates of eyeblink conditioning: Acquisition and retention. Learning \& Memory, 10, 427455 .

Collins, D. R., \& Pare, D. (2000). Differential fear conditioning induces reciprocal changes in the sensory responses of lateral amygdala neurons to the CS + and CS -. Learning \& Memory, 7, 97-103.

Davis, M. (1992). The role of the amygdala in fear and anxiety. Annual Review of Neuroscience, 15, 353-375.

Everitt, B. J., Cardinal, R. N., Parkinson, J. A., \& Robbins, T. W. (2003). Appetitive behavior: Impact of amygdala-dependent mechanisms of emotional learning. In P. Shinnick-Gallagher, A. Pitkänen, A. Shekhar, \& L. Cahill (Eds.), Annals of the New York Academy of Sciences: Vol. 985. The amygdala in brain function: Basic and clinical approaches (pp. 233-250). New York: New York Academy of Sciences.
Fanselow, M. S., \& Poulos, A. M. (2005). The neuroscience of mammalian associative learning. Annual Review of Psychology, 56, 207-234.

Gale, G. D., Anagnostaras, S. G., Godsil, B. P., Mitchell, S., Nozawa, T., Sage, J. R., et al. (2004). Role of the basolateral amygdala in the storage of fear memories across the adult lifetime of rats. Journal of Neuroscience, 24, 3810-3815.

Goosens, K. A., Hobin, J. A., \& Maren, S. (2003). Auditory-evoked spike firing in the lateral amygdala and Pavlovian fear conditioning: Mnemonic code or fear bias? Neuron, 40, 1013-1022.

Goosens, K. A., \& Maren, S. (2001). Contextual and auditory fear conditioning are mediated by the lateral, basal, and central amygdaloid nuclei in rats. Learning \& Memory, 8, 148-155.

Goosens, K. A., \& Maren, S. (2003). Pretraining NMDA receptor blockade in the basolateral complex, but not the central nucleus, of the amygdala prevents savings of conditional fear. Behavioral Neuroscience, 117, $738-750$.

Kapp, B. S., Frysinger, R. C., Gallagher, M., \& Haselton, J. R. (1979). Amygdala central nucleus lesions: Effect on heart rate conditioning in the rabbit. Physiology \& Behavior, 23, 1109-1117.

Killcross, S., Robbins, T. W., \& Everitt, B. J. (1997, July 24). Different types of fear-conditioned behaviour mediated by separate nuclei within amygdala. Nature (London), 388, 377-380.

LeDoux, J. E. (2000). Emotion circuits in the brain. Annual Review of Neuroscience, 23, 155-184.

Lee, T., \& Kim, J. J. (2004). Differential effects of cerebellar, amygdalar, and hippocampal lesions on classical eyeblink conditioning in rats. Journal of Neuroscience, 24, 3242-3250.

Maren, S. (1998). Overtraining does not mitigate contextual fear conditioning deficits produced by neurotoxic lesions of the basolateral amygdala. Journal of Neuroscience, 18, 3088-3097.

Maren, S. (1999a). Long-term potentiation in the amygdala: A mechanism for emotional learning and memory. Trends in Neurosciences, 22, 561567.

Maren, S. (1999b). Neurotoxic basolateral amygdala lesions impair learning and memory but not the performance of conditional fear in rats. Journal of Neuroscience, 19, 8696-8703.

Maren, S. (2000). Auditory fear conditioning increases CS-elicited spike firing in lateral amygdala neurons even after extensive overtraining. European Journal of Neuroscience, 12, 4047-4054.

Maren, S. (2001). Neurobiology of Pavlovian fear conditioning. Annual Review of Neuroscience, 24, 897-931.

Maren, S., Poremba, A., \& Gabriel, M. (1991). Basolateral amygdaloid multi-unit neuronal correlates of discriminative avoidance learning in rabbits. Brain Research, 549, 311-316.

Maren, S., \& Quirk, G. J. (2004). Neuronal signalling of fear memory. Nature Reviews Neuroscience, 5, 844-852.

McGaugh, J. L. (2004). The amygdala modulates the consolidation of memories of emotionally arousing experiences. Annual Review of Neuroscience, 27, 1-28.

Muramoto, K., Ono, T., Nishijo, H., \& Fukuda, M. (1993). Rat amygdaloid neuron responses during auditory discrimination. Neuroscience, 52, $621-636$

Nader, K., Majidishad, P., Amorapanth, P., \& LeDoux, J. E. (2001). Damage to the lateral and central, but not other, amygdaloid nuclei prevents the acquisition of auditory fear conditioning. Learning \& Memory, 8, 156-163.

Pare, D., Quirk, G. J., \& Ledoux, J. E. (2004). New vistas on amygdala networks in conditioned fear. Journal of Neurophysiology, 92(1), 1-9.

Pascoe, J. P., \& Kapp, B. S. (1985). Electrophysiological characteristics of amygdaloid central nucleus neurons during Pavlovian fear conditioning in the rabbit. Behavioural Brain Research, 16, 117-133.

Pelletier, J. G., Likhtik, E., Filali, M., \& Pare, D. (2005). Lasting increases in basolateral amygdala activity after emotional arousal: Implications for 
facilitated consolidation of emotional memories. Learning \& Memory, 12, 96-102.

Poremba, A., \& Gabriel, M. (1999). Amygdala neurons mediate acquisition but not maintenance of instrumental avoidance behavior in rabbits. Journal of Neuroscience, 19, 9635-9641.

Quirk, G. J., Armony, J. L., \& LeDoux, J. E. (1997). Fear conditioning enhances different temporal components of tone-evoked spike trains in auditory cortex and lateral amygdala. Neuron, 19, 613-624.

Quirk, G. J., Repa, C., \& LeDoux, J. E. (1995). Fear conditioning enhances short-latency auditory responses of lateral amygdala neurons: Parallel recordings in the freely behaving rat. Neuron, 15, 1029-1039.

Repa, J. C., Muller, J., Apergis, J., Desrochers, T. M., Zhou, Y., \& LeDoux, J. E. (2001). Two different lateral amygdala cell populations contribute to the initiation and storage of memory. Nature Neuroscience, 4, 724-731.
Richardson, R. T., \& Thompson, R. F. (1984). Amygdaloid unit activity during classical conditioning of the nictitating membrane response in rabbit. Physiology \& Behavior, 32, 527-539.

Rorick-Kehn, L. M., \& Steinmetz, J. E. (2005). Amygdalar unit activity during three learning tasks: Eyeblink classical conditioning, Pavlovian fear conditioning and signaled avoidance conditioning. Behavioral Neuroscience, 119, 1254-1276.

Uwano, T., Nishijo, H., Ono, T., \& Tamure, R. (1995). Neuronal responsiveness to various sensory stimuli, and associative learning in the rat amygdala. Neuroscience, 68, 339-361.

Received July 15, 2005

Revision received July 19, 2005

Accepted July 19, 2005 